\title{
Florbetaben PET in the Early Diagnosis of Alzheimer's Disease: A Discrete Event Simulation to Explore Its Potential Value and Key Data Gaps
}

\author{
Shien Guo, ${ }^{1}$ Denis Getsios, ${ }^{1}$ Luis Hernandez, ${ }^{1}$ Kelly Cho, ${ }^{2,3}$ Elizabeth Lawler, ${ }^{2,3,4}$ \\ Arman Altincatal, ${ }^{2,5}$ Stephan Lanes, ${ }^{1}$ and Michael Blankenburg ${ }^{6}$ \\ ${ }^{1}$ United BioSource Corporation, Health Economics-Modeling and Simulation, 430 Bedford Street, Suite 300, Lexington, \\ MA 02420, USA \\ ${ }^{2}$ Massachusetts Veterans Epidemiology Research and Information Center (MAVERIC) and VA Boston Healthcare System, \\ 1400 VFW Parkway, West Roxbury, MA 02132, USA \\ ${ }^{3}$ Division of Aging, Department of Medicine, Brigham and Women's Hospital, Harvard Medical School, 1620 Tremont Street, \\ Boston, MA 02120, USA \\ ${ }^{4}$ Boston University School of Public Health, 715 Albany Street, Boston, MA 02118, USA \\ ${ }^{5}$ United BioSource Corporation, Biostatistics, 430 Bedford Street, Suite 300, Lexington, MA 02420, USA \\ ${ }^{6}$ Bayer HealthCare Pharmaceuticals, Global Market Access - General Medicine, 178 Müllerstraße, 13353 Berlin, Germany
}

Correspondence should be addressed to Shien Guo, shien.guo@unitedbiosource.com

Received 26 July 2012; Accepted 5 October 2012

Academic Editor: Francesco Panza

Copyright () 2012 Shien Guo et al. This is an open access article distributed under the Creative Commons Attribution License, which permits unrestricted use, distribution, and reproduction in any medium, provided the original work is properly cited.

The growing understanding of the use of biomarkers in Alzheimer's disease (AD) may enable physicians to make more accurate and timely diagnoses. Florbetaben, a beta-amyloid tracer used with positron emission tomography (PET), is one of these diagnostic biomarkers. This analysis was undertaken to explore the potential value of florbetaben PET in the diagnosis of AD among patients with suspected dementia and to identify key data that are needed to further substantiate its value. A discrete event simulation was developed to conduct exploratory analyses from both US payer and societal perspectives. The model simulates the lifetime course of disease progression for individuals, evaluating the impact of their patient management from initial diagnostic work-up to final diagnosis. Model inputs were obtained from specific analyses of a large longitudinal dataset from the New England Veterans Healthcare System and supplemented with data from public data sources and assumptions. The analyses indicate that florbetaben PET has the potential to improve patient outcomes and reduce costs under certain scenarios. Key data on the use of florbetaben PET, such as its influence on time to confirmation of final diagnosis, treatment uptake, and treatment persistency, are unavailable and would be required to confirm its value.

\section{Introduction}

Alzheimer's disease $(\mathrm{AD})$ is a fatal and progressive neurodegenerative disorder that affects millions of people worldwide [1]. It is currently the sixth leading cause of death in the United States (US), with about 80,000 deaths associated with $\mathrm{AD}$ in 2009 [2], and has imposed substantial burden on patients, their caregivers, medical care payers, and society as a whole [3]. With the baby-boomer generation aging, the economic and humanistic burdens caused by $\mathrm{AD}$ are expected to grow considerably if effective interventions cannot be discovered in time.
There is still no cure for AD. Cholinesterase inhibitors and memantine are the only major pharmacological treatments currently available to slow the symptoms associated with disease progression. Clinical studies have demonstrated modest benefits of these treatments in improving the symptoms related to $\mathrm{AD}[4,5]$. Prior studies have also indicated that identifying patients with $\mathrm{AD}$ and treating them at an early stage could result in cost savings and health benefits compared with treating them at a later stage due to the absence of early assessment [6, 7]. Current diagnostic tools in the early diagnosis of $\mathrm{AD}$, however, are imprecise and detect the disease only based on the symptoms. Misdiagnosis 
and delayed diagnosis of $\mathrm{AD}$, therefore, have been commonly reported [8-10].

The current diagnosis of $\mathrm{AD}$ is mainly based on clinical grounds according to the guidelines developed jointly by the National Institute of Neurological and Communicative Disorders and Stroke and the Alzheimer's Disease and Related Disorders Association (NINCDS-ADRDA). Although sensitive for $\mathrm{AD}$, the NINCDS-ADRDA guidelines have poor specificity [11], which could lead to a great number of false positive cases and, consequently, unnecessary treatment. The growing knowledge in the use of biomarkers of AD pathology is very likely to provide physicians with new tools to not only improve the differential diagnosis of $\mathrm{AD}$ but also identify $\mathrm{AD}$ at an earlier stage and even before symptoms occur. The International Working Group for New Research Criteria for the Diagnosis of $\mathrm{AD}$ has recently revised the definition of $\mathrm{AD}$ to include both the predementia (or prodromal $\mathrm{AD}$ ), referring to the early symptomatic phase of $\mathrm{AD}$ that is still not sufficiently severe to affect instrumental activities of daily living, and dementia (or AD dementia) phases. It has also recommended that the diagnosis of $\mathrm{AD}$ relies on a dual clinicobiological process that entails the evidence of both specific cognitive impairments and biomarkers of $\mathrm{AD}$ pathology that can include retention of amyloid tracers on positron emission tomography (PET); cerebral spinal fluid (CSF) beta-amyloid, total tau, and phospho-tau; medial temporal lobe atrophy (MTA) on magnetic resonance imaging (MRI); and/or temporal/parietal hypometabolism on fluorodeoxyglucose (FDG-) PET [12].

Florbetaben, a beta-amyloid tracer, is one of the novel diagnostic tools that can be used to detect neuropathological changes related to $\mathrm{AD}$ in vivo. It binds to betaamyloid plaques and can be detected using a PET scan. The predictive values of florbetaben PET are currently under a phase III assessment, but preliminary phase II data have shown promising results in discriminating $\mathrm{AD}$ from other dementias and healthy controls [13-15]. The implications of this diagnostic tool in detection, diagnosis, and treatment of $\mathrm{AD}$ in actual clinical practice could be substantial once it is approved for use. However, due to increasing health resource constraints, the widespread use of florbetaben PET in the future clinical practice would depend not only on its clinical value but also its economic impact. Thus, the purposes of this study were to develop an early exploratory economic model assessing the potential clinical and economic value of florbetaben PET in the diagnosis of AD and to identify key value drivers as well as data gaps, which will direct the future research to support the future economic assessment of this technology. The design of this model and the findings from this assessment could also be very useful to guide further research assessing the cost effectiveness of other biomarkers, in the diagnosis of $\mathrm{AD}$.

\section{Materials and Methods}

The model explores the potential clinical and economic consequences of using florbetaben PET in the usual diagnostic process for the diagnosis of $\mathrm{AD}$ from both the US payer and societal perspectives. Usual diagnostic care refers to a period of diagnostic work-up during which specific diagnostic tests are performed over a series of medical visits to obtain the needed information for confirmation of a specific type of dementia diagnosis. Different combinations of diagnostic tests and assessments, along with other tests of pathophysiological (e.g., amyloid tracers and total tau) and/or topographical makers (e.g., FDG and MTA), received by patients during the diagnostic work-up period, could have different predictive values in terms of differentiating $\mathrm{AD}$ from other forms of dementia; there are numerous possible combinations of the tests and assessments that can be received by patients during the work-up period. Thus, for the purpose of simplicity and availability of the existing data to inform the predictive values of each specific combination of tests received by patients, the possible combinations of the diagnostic tests in this model were categorized into the following algorithms: clinical guidelines alone (e.g., NINCDS-ADRDA) and clinical guidelines plus one of the following tests: (1) MRI of MTA, (2) computerized tomography (CT) of MTA, (3) FDG-PET, (4) single photon emission computer tomography (SPECT), (5) CSF of beta-amyloid, total tau, phospho-tau, or beta-amyloid plus total tau, and (6) PET of florbetaben beta-amyloid tracers. Patients in the nonflorbetaben group (or called usual diagnostic care group hereafter) can be proportionally assigned to any of these diagnostic algorithms, except for the clinical guidelines plus florbetaben PET, to obtain an aggregate measure of sensitivity and specificity associated with the diagnostic algorithms assigned.

This model was implemented as an individual patient simulation using discrete event simulation (DES) [24]. This modelling technique was selected mainly due to its capability to track events experienced by patients over the course of simulation and to use them, along with other relevant patient and disease characteristics, to predict the course of disease progression over time. This feature, along with other advantages, has led to increasing use of DES in assessing the cost effectiveness of treatments in patients with $\mathrm{AD}$ $[7,25,26]$.

2.1. Model Concept. Figure 1 shows the simplified schematic representation of the model concept. The target model population includes patients with some form of dementia who present to doctor offices for the first time, and those with cognitive problems due to other illnesses, such as depression or schizophrenia, were not considered in this analysis. Patients could make their initial doctor visit in either the predementia or dementia phase of the disease. The latter can be further divided into several levels of severity based on the Mini-Mental State Examination (MMSE) scores. Patients in the dementia phase are assigned an underlying pathological cause of dementia, which can be one of the following types of dementia: $\mathrm{AD}$, mixed $\mathrm{AD}$, vascular dementia $(\mathrm{VaD})$, Lewy body dementia (LBD), frontotemporal dementia (FTD), and mixed non-AD. Those in the predementia phase are also assigned an underlying cause of either prodromal $\mathrm{AD}$ or nonprodromal $\mathrm{AD}$, which could be any of the non$\mathrm{AD}$ dementia listed above if the patient progresses to the 


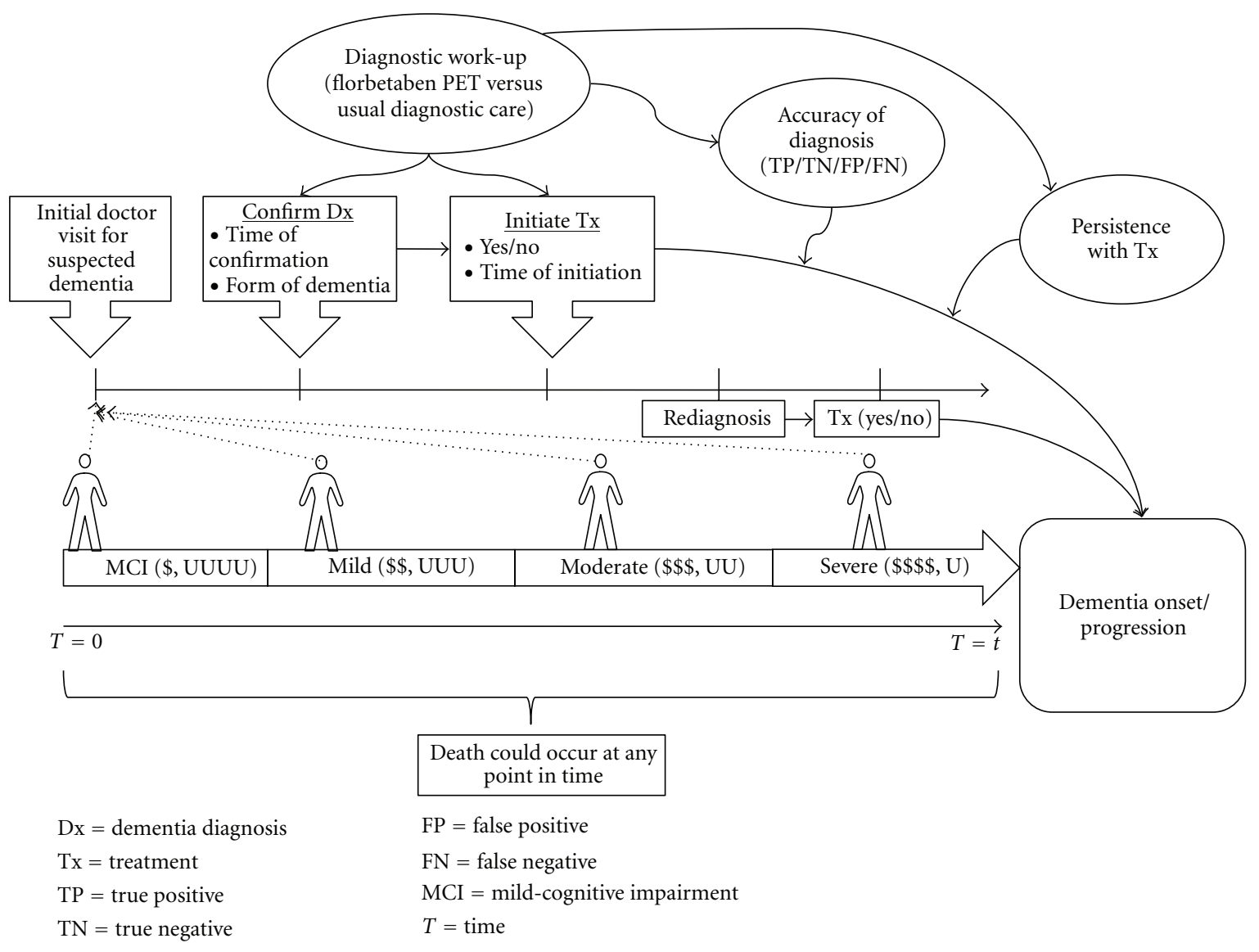

FIGURE 1: Schematic representation of the model concept.

dementia phase. Relevant patient and disease characteristics, such as age, gender, race, location of care, baseline severity, and comorbidities, are also assigned to each patient at the beginning of the simulation conditional on their underlying cause.

Each patient in the model undergoes a period of diagnostic work-up following their initial doctor visit. At the end of the diagnostic work-up period, all the predementia patients are assumed to be correctly confirmed with a predementia diagnosis, and those assigned to the florbetaben group are given a PET scan, with dementia treatment, such as cholinesterase inhibitors and memantine, initiated for those with a positive $A D$ result. For those assigned to the usual diagnostic care group, all or some proportion of the patients may receive dementia treatment without screening after their predementia diagnosis is confirmed.

For the dementia patients, all patients are confirmed with a specific type of dementia at the end of diagnostic workup, but the correctness of the diagnosis is dependent on the predictive values of the diagnostic algorithm assigned to the patient. Dementia treatment is initiated to all dementia patients in the florbetaben group with a positive AD result, but not to those with a negative result at the time of diagnosis confirmation. For those in the usual diagnostic care group, some dementia patients also have a chance to receive dementia treatment at the time of confirmation, depending on the result of diagnosis. Patients with a positive AD result but not treated at the time of confirmation could receive dementia treatment at a later time.

Dementia treatment may delay progression to the dementia phase for patients with predementia. When a patient develops dementia, treatment may slow progression to a more severe stage of the disease, as well as need for institutional care, but the effect of treatment could be negatively impacted by misdiagnosis and nonpersistence with treatment. Disease progression in this model was modelled through the interrelated changes in 3 domains over time: cognition, using MMSE scale; behaviour, using the Neuropsychiatric Inventory (NPI) scale; function, using both the activities of daily living (ADL) and instrumental activities of daily living (IADL) scales. In this model, it is assumed that only those with prodromal $\mathrm{AD}, \mathrm{AD}$, or mixed $\mathrm{AD}$ as the underlying cause of dementia would benefit from the treatment with cholinesterase inhibitors or memantine. Treatment initiated in patients with non-AD would only have an impact on treatment costs.

Disease progression continues during the diagnostic work-up period. Shortening the time required to correctly confirm a diagnosis would allow appropriate treatments to be initiated at an earlier stage of the disease and thus could 
result in greater health benefits at lower costs. Use of florbetaben PET in this model could directly influence 4 major areas: (1) time required to confirm a diagnosis, (2) accuracy of diagnosis, (3) proportion of patients receiving appropriate dementia treatments at confirmation, and (4) persistence with treatment. Each of these impacts is associated with specific clinical and economic consequences. Finally, this model allows those who are misdiagnosed to be correctly rediagnosed at a later time and receive treatment. Death can occur at any point in time, and is dependent on patient age, gender, underlying cause of dementia, and stage of the disease (i.e., predementia and dementia phases). A simplified model flow diagram showing how patients are simulated is displayed and explained in Appendix A.

2.2. Data Sources. The primary data source used to populate this model was based on the administrative databases from New England Veterans Healthcare System (VISN 1) from January 1, 2002 through December 31, 2009 (fiscal years 2002-2009). Specific analyses from the VA VISN 1 data were performed to obtain the majority of the model inputs. Detailed information on the VA VISN 1 data can be seen in Appendix B. Model inputs which could not be obtained from the VA VISN 1 data were supplemented with data from literature, public databases, and assumptions.

2.2.1. Model Settings. A reference-case analysis was performed based on 1,000 simulated patients per group per run for a total of 10 replications. The model time horizon for the reference-case analysis was lifetime, which is commonly used for the assessment in this therapeutic area. Costs and benefits were discounted at 3\% per annum [27]. Additionally, disease severity was divided into five levels based on the ranges of MMSE scores previously used by the United Kingdom National Institute for Health and Clinical Excellence [28].

2.2.2. Patient and Disease Characteristics at Baseline. Data used to create the model population in the simulation were mainly obtained from the VA VISN 1 data, supplemented with data from literature. Among the patients with a confirmed diagnosis, $68 \%$ of them were confirmed with a dementia diagnosis and $32 \%$ with a predementia diagnosis. For those with a dementia diagnosis, $67 \%$ had a confirmed diagnosis of $\mathrm{AD}$ or mixed $\mathrm{AD}, 28 \%$ had $\mathrm{a} \mathrm{VaD}$, and the remaining $5 \%$ had other dementia diagnoses, such as LBD, FTD, and mixed non-AD. For those diagnoses with predementia, 65\% were assumed to have prodromal $\mathrm{AD}$, which was estimated based on a chart review of a subset of these patients. The mean age at initial diagnosis was about 78 years for patients with predementia and 82 years for patients with dementia. Data used to assign gender to the model populations (about 30\% male) were obtained from literature [29-31] rather than from the VA VISN 1 data as almost all patients in the study cohort were male.

Baseline MMSE scores were obtained from a chart review of a subset $(n=229)$ of the VA VISN 1 study cohort, indicating that more than $60 \%$ and $80 \%$ of the $\mathrm{AD}$ and nonAD patients, respectively, had the MMSE scores above 20 at initial diagnosis. As the NPI, ADL, and IADL scores were not available from the VA VISN 1 data, assumptions were made to assign the baseline scores for these measures consistent with the distribution of severity level based on the baseline MMSE scores.

\subsubsection{Diagnostic Algorithms and Corresponding Predictive} Values. The proportions of patients in the usual diagnostic care group undergoing a specific diagnostic algorithm were obtained from the VA VISN 1 data (Table 1). Table 1 also shows sensitivity and specificity by severity of the disease for each diagnostic algorithm. Data, except for florbetaben PET, were from a recent meta-analysis conducted by Bloudek and colleagues [11]. The sensitivity and specificity of florbetaben PET were supplied by the manufacturer of florbetaben tracers based on its internal analyses of preliminary phase II data as well as published data from other amyloid tracers [13-15].

2.2.4. Time to Confirmation of Diagnosis. The amount of time taken to confirm a specific type of dementia diagnosis from the initial office visit under usual diagnostic care was predicted using parametric equations derived from the VA VISN 1 data. Table 2 shows the equation for each diagnosis. Predictors with a positive coefficient indicate a longer time to confirmation of diagnosis as the values of the predictors increase and vice versa. The VA VISN 1 data show that the average time to diagnosis confirmation was about 5.1 months for $\mathrm{AD}$ or mixed $\mathrm{AD}, 6.1$ months for $\mathrm{VaD}, 5.7$ months for other non-AD, and 5.5 months for predementia diagnosis. In the reference-case analysis, use of a florbetaben PET was assumed to lead to a $50 \%$ reduction in time to diagnosis confirmation under usual diagnostic care. As the evidence to support this still does not exist, extensive sensitivity analyses were used to assess the impact of these assumptions on predicted outcomes.

2.2.5. Dementia Treatment. Dementia medications can be initiated at either the time of diagnosis confirmation or a later time for those patients in the usual diagnostic care group (Table 3 ). The majority of the patients were treated with donepezil based on the VA VISN 1 data. For patients in the usual diagnostic care group who were not treated at diagnosis confirmation, their time to treatment initiation was predicted using the parametric equations derived from the VA VISN 1 data (Table 2), indicating a median time of 28 months to treatment initiation for the dementia cohort and 42 months for the predementia cohort.

Patients on any dementia treatment may discontinue over time. Table 2 shows the parametric equations used to predict the time to discontinuation based on the VA VISN 1 data, indicating a median time of 36 months to treatment discontinuation for patients with dementia and 42 months for patients with predementia. The latter included the duration of treatment during the dementia phase. In the reference-case analysis, it was assumed that use of florbetaben PET could reduce the risk of discontinuation by $50 \%$. This assumption, also tested in the sensitivity analyses, 
TABLE 1: Distribution of diagnostic algorithms and corresponding accuracy by severity (based on MMSE).

\begin{tabular}{|c|c|c|c|c|c|c|}
\hline \multirow{2}{*}{ Diagnostic algorithm } & \multicolumn{2}{|c|}{$\%$} & \multicolumn{2}{|c|}{ Mild } & \multicolumn{2}{|c|}{ Moderate and severe } \\
\hline & Usual care & Florbetaben & Sensitivity & Specificity & Sensitivity & Specificity \\
\hline Clinical guidelines only & $73 \%$ & $0 \%$ & $87 \%$ & $59 \%$ & $77 \%$ & $73 \%$ \\
\hline \multicolumn{7}{|l|}{ Clinical guidelines with } \\
\hline MRI of MTA & $8 \%$ & $0 \%$ & $82 \%$ & $66 \%$ & $85 \%$ & $80 \%$ \\
\hline CT of MTA & $19 \%$ & $0 \%$ & $80 \%$ & $87 \%$ & $80 \%$ & $87 \%$ \\
\hline FDG-PET & $0 \%$ & $0 \%$ & $91 \%$ & $75 \%$ & $91 \%$ & $86 \%$ \\
\hline SPECT & $0 \%$ & $0 \%$ & $79 \%$ & $81 \%$ & $68 \%$ & $86 \%$ \\
\hline CSF $\mathrm{A} \beta 1-42$ & $0 \%$ & $0 \%$ & $72 \%$ & $75 \%$ & $74 \%$ & $79 \%$ \\
\hline CSF A $\beta 1-42+$ Ttau & $0 \%$ & $0 \%$ & $86 \%$ & $64 \%$ & $84 \%$ & $72 \%$ \\
\hline CSF Ttau & $0 \%$ & $0 \%$ & $77 \%$ & $73 \%$ & $82 \%$ & $71 \%$ \\
\hline CSF Ptau & $0 \%$ & $0 \%$ & $77 \%$ & $73 \%$ & $82 \%$ & $78 \%$ \\
\hline Florbetaben PET & $0 \%$ & $100 \%$ & $90 \%$ & $90 \%$ & $90 \%$ & $90 \%$ \\
\hline
\end{tabular}

MRI: magnetic resonance imaging; MTA: medial temporal lobe atrophy; CT: computer tomography; PET: positron emission tomography; FDG: fluorodeoxyglucose; SPECT: single photon emission computer tomography; CSF: cerebral spinal fluid.

is based on the rationale that the improved accuracy of florbetaben PET may increase physicians' and patients' level of confidence in the diagnosis and therefore encourage them to persist longer with treatment.

In this model, dementia treatment can also be forced to stop under some conditions specified by the users. In the reference-case analysis, patients with dementia were allowed to receive dementia treatment for lifetime as long as their MMSE scores were greater than 10; patients with predementia were assumed to receive treatment for no longer than five years if the patient did not covert to the dementia phase.

2.2.6. Disease Progression. Patients with predementia may convert to the dementia phase at a later time. Data on time to conversion, also from the VA VISN 1 data, were fitted to a Weibull function (Table 2). Based on the VA VISN 1 data, the median time to conversion was about 35 months. In the reference-case analysis, it was assumed that dementia treatment during the predementia phase would reduce the risk of conversion by $50 \%$ among those patients with prodromal $\mathrm{AD}$, although the evidence to support such clinical benefits remains uncertain. This assumption was made to also assess the potential benefits of using florbetaben PET to screen predementia patients for treatment if a new effective treatment becomes available in the near future. Treatment for those patients with nonprodromal AD would have no clinical benefit but would have a cost impact.

Disease progression for patients with $\mathrm{AD}$ or mixed $\mathrm{AD}$ was modelled based on the interrelated changes in MMSE, NPI, ADL, and IADL over time. Data used to simulate the disease progression were based on the predictive equations (Table 2) obtained from a study conducted by Getsios and colleagues [25]. On the other hand, disease progression for patients with non-AD dementia was simulated only based on changes in MMSE over time due to lack of data on NPI, ADL, and IADL. The same equation from Getsios and colleagues was used without any adjustment as few studies have shown that patients with other non-AD dementia, except for FTD, have similar rates of decline in cognition to those with $\mathrm{AD}$ [32-35]. For patients with FTD, the annual rate of change in MMSE scores was adjusted by -4.4 points [34].

2.2.7. Time to Institutional Care. Two Weibull equations derived from the VA VISN 1 data were used to predict the time to institutional care for dementia and predementia patients (Table 2). Age was the only predictor in the equation for patients with predementia, indicating that the older patients are more likely to need institutional care than younger patients. On the other hand, predictors in the equation for patients with dementia include type of dementia diagnosis, time to confirmed diagnosis, and use of any dementia medication. Patients diagnosed with $\mathrm{AD}$ were more likely to need institutional care than patients diagnosed with other types of dementia; a longer time to confirmation of diagnosis was associated with a shorter time to institutional care; the and use of any dementia medication was associated with a longer time to institutional care.

2.2.8. Time to Death. Time to death was predicted using two gender-specific Gompertz functions derived from the US Life Table based on patients age 55 years and above (Table 2) [36]. These baseline risks were adjusted with hazard ratios of 1.48 for patients with predementia, 2.84 for patients with $\mathrm{AD}$ or mixed $\mathrm{AD}$, and 2.69 for patients with non-AD dementia [37].

2.2.9. Costs and Resource Uses. Cost inputs and their corresponding sources are shown in Table 4. Cost items considered in this model included costs of diagnostic workup, imaging and biomarker tests, dementia medications, and medical and nonmedical care for predementia and dementia, including caregiver time. All cost inputs used 2011 values. Detailed information on cost inputs can be viewed in Appendix C.

2.2.10. Utilities. The model estimates utilities for both patients and their caregivers. Health utilities for patients with 
TABLE 2: Equations for prediction of time to events and disease progression.

\begin{tabular}{|c|c|c|c|}
\hline Equation & Coefficient and predictor & SD/shape & Distribution \\
\hline \multicolumn{4}{|c|}{ Time to confirmation of a diagnosis } \\
\hline $\mathrm{AD}$ or mixed $\mathrm{AD}$ & $4.571+0.327$ Male +0.252 MixedAD $-0.353 \mathrm{CKD}$ & 0.965 & Lognormal \\
\hline $\mathrm{VaD}$ & $4.529+0.158$ Diabetes +0.203 Hypertension +0.385 Stroke & 1.005 & Lognormal \\
\hline Other non-AD dementia & $6.558-0.029$ Age +1.554 Stroke + 0.654 LBD + 0.400 FTD & 0.896 & Lognormal \\
\hline Predementia & $3.981+0.009$ Age $-0.243 \mathrm{CKD}-0.179 \mathrm{CVD}$ & 0.994 & Lognormal \\
\hline \multicolumn{4}{|l|}{$\begin{array}{l}\text { Time to treatment initiation if not } \\
\text { started at diagnosis }\end{array}$} \\
\hline Dementia & $\begin{array}{l}7.149-0.022 \text { Age }+1.056 \mathrm{VaD}+2.091 \text { Other non-AD }+0.004 \\
\text { (time, in days, to confirmed diagnosis) }\end{array}$ & 1.517 & Lognormal \\
\hline Predementia & $18.781-0.150$ Age & 3.996 & Lognormal \\
\hline \multicolumn{4}{|l|}{ Time to treatment discontinuation } \\
\hline Dementia & $7.487-0.0008$ (time to confirmed diagnosis) & 0.922 & Weibull \\
\hline Predementia & $7.122+0.443$ (conversion to dementia) & 1.131 & Weibull \\
\hline Time to conversion to dementia & Scale $=0.0212$ & 0.952 & Weibull \\
\hline Rate of change in MMSE ${ }^{a}$ & $\begin{array}{l}5.4663-0.4200 \mathrm{PM} 1-0.0042 \mathrm{PM} 2+0.1415 \mathrm{PM} 3- \\
0.079 \text { PrevRate }+0.07474 \text { Age }+\delta_{i}\end{array}$ & N/A & N/A \\
\hline Rate of change in $\mathrm{NPI}^{\mathrm{b}}$ & 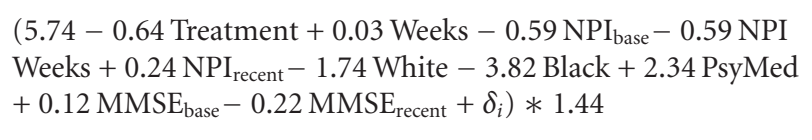 & N/A & N/A \\
\hline Rate of change in ADL & $\begin{array}{l}1.35-0.81 \text { Treatment }+0.06 \text { Weeks }-0.79 \mathrm{ADL}_{\text {base }}+ \\
0.71 \mathrm{IADL}_{\text {previous }}+0.12 \mathrm{MMSE}_{\text {base }}+0.09 \text { Age }+0.81 \text { PsyMed }- \\
3.05 \text { Black }-0.49 \mathrm{MMSE}_{\text {recent }}+\delta_{i}\end{array}$ & N/A & N/A \\
\hline Rate of change in IADL & $\begin{array}{l}1.27+0.63 \text { Treatment }+0.17 \text { Weeks }-0.06 \text { Treatment } * \text { Weeks } \\
-0.84 \mathrm{IADL}_{\text {base }}-0.002 \mathrm{IADL}_{\text {base }} * \text { Weeks }+0.84 \mathrm{IADL}_{\text {previous }}- \\
0.67 \mathrm{Male}+0.20 \mathrm{MMSE}_{\text {base }}-0.28 \mathrm{MMSE}_{\text {recent }}-0.16 \mathrm{ADL}_{\text {base }}+ \\
0.18 \mathrm{ADL}_{\text {recent }}+\delta_{i}\end{array}$ & N/A & N/A \\
\hline \multicolumn{4}{|l|}{ Time to institutional care } \\
\hline Dementia & $\begin{array}{l}9.883-0.02 \text { Age }+0.295 \mathrm{VaD}+1.154 \text { Other non-AD }- \\
0.001 \text { Time to confirmed diagnosis }+1.079 \text { Dementia treatment }\end{array}$ & 0.933 & Weibull \\
\hline Predementia & $11.469-0.028$ Age & 1.373 & Weibull \\
\hline \multicolumn{4}{|l|}{ Time to death } \\
\hline Male & Scale $=-9.697$ & 0.087 & Gompertz \\
\hline Female & Scale $=-10.787$ & 0.097 & Gompertz \\
\hline Patient utility & $\begin{array}{l}0.408+0.010 \text { MMSE }-0.004 \text { NPI }-0.159 \text { Institutionalized + } \\
0.051 \text { Living with Caregiver }\end{array}$ & N/A & N/A \\
\hline Caregiver utility & $\begin{array}{l}0.90-0.003 \text { Age }_{\mathrm{CG}}+0.03 \text { Male }_{\mathrm{CG}}+0.001 \text { Male }-0.001 \mathrm{NPI}- \\
0.001 \mathrm{ADL}-0.0004 \mathrm{IADL}-0.01 \text { PsyMed }\end{array}$ & $\mathrm{N} / \mathrm{A}$ & N/A \\
\hline
\end{tabular}

SD: standard deviation; AD: Alzheimer's disease; VaD: vascular dementia; LBD: Lewy body dementia; LTD: frontotemporal dementia; CKD: chronic kidney disease; CVD: cerebrovascular disease; MMSE: mini-mental state examination; NPI: neuropsychiatric inventory; ADL: activities of daily living; IADL: instrumental activities of daily living, CG: caregiver.

${ }^{\text {a }}$ PM represents patients' previous MMSE measurement, partitioned over the scale of MMSE. PrevRate is the patients' last known rate of decline. Age represents patients' age at baseline. $\delta_{i}$ represents a random intercept parameter.

${ }^{\mathrm{b}}$ Treatment is dementia medication, Weeks represents weeks of followup in the simulation, NPI $\mathrm{base}_{\text {is }}$ the patient's baseline NPI, and NPI ${ }_{\text {recent }}$ is the patient's last NPI. White and Black are dummy variables for race, PsyMed is a dummy variable for patients on psychiatric medications at baseline, MMSE $\mathrm{base}_{\mathrm{bepresents}}$ the patient's MMSE at baseline, and MMSE ${ }_{\text {recent }}$ represents the patient's current MMSE. 
TABLE 3: Model parameters for treatments.

\begin{tabular}{|c|c|c|c|c|c|}
\hline \multirow{2}{*}{ Parameter } & \multicolumn{2}{|c|}{ Dementia } & \multicolumn{2}{|c|}{ Predementia } & \multirow{2}{*}{ Data source } \\
\hline & Usual care & Florbetaben & Usual care & Florbetaben & \\
\hline $\begin{array}{l}\% \text { of patients receiving dementia medication at } \\
\text { diagnosis }\end{array}$ & N/A & N/A & $28 \%$ & N/A & VA VISN 1 and user specification \\
\hline If $\mathrm{Dx}=\mathrm{AD}+$ & $77 \%$ & $100 \%$ & N/A & $100 \%$ & \\
\hline If $\mathrm{Dx}=$ non- $\mathrm{AD}$ & $67 \%$ & $0 \%$ & N/A & $0 \%$ & \\
\hline Distribution of dementia medication & $\mathrm{Dx}=\mathrm{AD}+$ & $\mathrm{Dx}=$ Non- $\mathrm{AD}$ & & & VA VISN 1 \\
\hline Donepezil & $63 \%$ & $66 \%$ & & $76 \%$ & \\
\hline Galantamine & $25 \%$ & $9 \%$ & & $6 \%$ & \\
\hline Rivastigmine & $5 \%$ & $4 \%$ & & $1 \%$ & \\
\hline Memantine & $7 \%$ & $21 \%$ & & $7 \%$ & \\
\hline $\begin{array}{l}\text { Maximum dementia treatment duration } \\
\text { allowed, years }\end{array}$ & \multicolumn{2}{|c|}{ Life time } & \multicolumn{2}{|r|}{5} & User specification \\
\hline $\begin{array}{l}\text { Stopping dementia medication if MMSE score } \\
\text { is below } 10\end{array}$ & \multicolumn{2}{|r|}{ Yes } & \multicolumn{2}{|c|}{$\mathrm{N} / \mathrm{A}$} & User specification \\
\hline
\end{tabular}

AD: Alzheimer's disease; Dx: dementia diagnosis; MMSE: mini-mental state examination; N/A: not applicable.

$\mathrm{AD}$ were estimated based on a published regression equation shown in Table 2 [38]. Health utilities for patients with non$\mathrm{AD}$ were estimated by adjusting 0.006 lower compared to their AD counterparts [39]. A utility weight of 0.82 was used for patients with predementia [40]. Caregiver utilities were predicted with an equation (Table 2 ) from a report by Getsios and colleagues [25].

\section{Results}

\subsection{Reference-Case Analyses}

3.1.1. At Baseline. Of the 1,000 simulated patients, $32 \%$ had predementia and $68 \%$ dementia, replicating the underlying input data. Of those patients with predementia, $65 \%$ had prodromal $\mathrm{AD}$ as the underlying cause. Patients with predementia had a mean age of 78 years, and mean scores of 27.5 on the MMSE, 2.5 on the NPI, and 10.1 on both ADL and IADL scales. On the other hand, for those with dementia, $67 \%$ had $\mathrm{AD}$ or mixed $\mathrm{AD}$ as the underlying cause, $28 \%$ had $\mathrm{VaD}$, and $5 \%$ had $\mathrm{LBD}$, FTD, or other mixed no-AD dementia with a mean age of 82 years and mean scores of 21.9 on the MMSE, 16.3 on the NPI, 29.7 on ADL, and 29.1 on IADL scales.

3.1.2. Predementia Cohort. The reference-case analysis shows that the average time to confirmation of predementia diagnosis was 4.64 months under usual diagnostic care, which was slightly lower than the time indicated by the VA VISN 1 data due to death and early conversion to dementia during the diagnostic work-up period, and 2.49 months with use of florbetaben PET.

Due to death and early conversion, only about $92 \%$ $(n=295)$ of the predementia patients in the florbetaben group received the scan. Of these, $62 \%$ had a positive result, consisting of $58 \%$ true positive cases and $4 \%$ false positive cases (i.e., patients had non-AD dementia, but misdiagnosed as having $\mathrm{AD}$ ), and thus received dementia treatment. For the remaining $38 \%$ of patients with a negative result, including $31 \%$ true negative and $7 \%$ false negative cases (i.e., patients had $\mathrm{AD}$, but misdiagnosed as having non-AD dementia), dementia treatment was not initiated. The florbetaben group had a mean life expectancy of 0.10 years longer than the usual diagnostic group (Table 5), which was due to the delay in conversion to dementia in which the risk of death was greater. On average, patients in the florbetaben group had better other clinical outcomes than patients in the usual diagnostic groups in terms of time staying in predementia phase, time to institutional care, time spent in severer stages of the disease, and caregiver time (Table 5). These resulted in net discounted cost savings of $\$ 12,374$ per patient over lifetime in direct medical care, $\$ 643$ in caregiver time, and $\$ 13,018$ in total cost. These savings were mainly due to reduction in several cost areas, with the greatest savings coming from reduced institutional care. Moreover, these clinical benefits also led to a net QALY gain (Table 5), making the use of florbetaben PET a dominant strategy in identifying and treating patients with prodromal $\mathrm{AD}$ under this referencecase scenario.

3.1.3. Dementia Cohort. The reference-case results for the dementia cohort are also shown in Table 5 . The average time to diagnosis confirmation was about 5.08 months with the usual diagnostic care group versus 2.66 months with the florbetaben group. Due to death, about $91 \%$ of the patients in the usual diagnostic care group and $95 \%$ in the florbetaben group completed the diagnostic work-up. More patients were misdiagnosed in the usual diagnostic care group, with $12 \%$ 
TABle 4: Cost inputs.

\begin{tabular}{|c|c|c|c|}
\hline Cost item & Value & Unit & Data source \\
\hline \multicolumn{4}{|l|}{ Diagnostic work-up } \\
\hline $\mathrm{AD}+$ & $\$ 5,120$ & Per year & \multirow{4}{*}{ VA VISN 1 and [16-18] } \\
\hline $\mathrm{VaD}$ & $\$ 5,885$ & Per year & \\
\hline Other non-AD & $\$ 6,638$ & Per year & \\
\hline Predementia & $\$ 6,187$ & Per year & \\
\hline \multicolumn{4}{|l|}{ Imaging and biomarker tests } \\
\hline MRI + MTA & $\$ 437$ & Per test & \multirow{6}{*}[16]{, manufacturer } \\
\hline $\mathrm{CT}+\mathrm{MTA}$ & $\$ 300$ & Per test & \\
\hline FDG-PET & $\$ 1,042$ & Per test & \\
\hline SPECT & $\$ 596$ & Per test & \\
\hline CSF & $\$ 304$ & Per test & \\
\hline Florbetaben PET & $\$ 2,300$ & Per test & \\
\hline \multicolumn{4}{|l|}{ Dementia medication } \\
\hline Donepezil & $\$ 7.79$ & Per day & \multirow{4}{*}[19]{} \\
\hline Galantamine & $\$ 6.36$ & Per day & \\
\hline Rivastigmine & $\$ 6.11$ & Per day & \\
\hline Memantine & $\$ 7.89$ & Per day & \\
\hline Medical care for predementia & $\$ 5,548$ & Per year & {$[20]$} \\
\hline \multicolumn{4}{|l|}{ Medical care for $\mathrm{AD}+$} \\
\hline Mild & $\$ 8,315$ & Per year & \multirow{5}{*}[20]{} \\
\hline Mildly moderate & $\$ 12,806$ & Per year & \\
\hline Moderate & $\$ 12,806$ & Per year & \\
\hline Moderately severe & $\$ 18,526$ & Per year & \\
\hline Severe & $\$ 23,227$ & Per year & \\
\hline \multicolumn{4}{|l|}{ Nonmedical care for AD+ } \\
\hline Mild & $\$ 154$ & Per year & \multirow{5}{*}[20]{} \\
\hline Mildly moderate & $\$ 3,692$ & Per year & \\
\hline Moderate & $\$ 12,166$ & Per year & \\
\hline Moderately severe & $\$ 14,209$ & Per year & \\
\hline Severe & $\$ 23,355$ & Per year & \\
\hline \multicolumn{3}{|c|}{$\%$ of additional cost of care for non- $\mathrm{AD}$ relative to $\mathrm{AD}$} & \multirow{3}{*}[21]{} \\
\hline $\mathrm{VaD}$ & $84 \%$ & & \\
\hline Other non-AD & $37 \%$ & & \\
\hline Institutional care & $\$ 373$ & Per day & {$[22]$} \\
\hline Caregiver time & $\$ 7.25$ & Per hour & {$[23]$} \\
\hline Caregiver burden for predementia & 2.10 & Hours per day & {$[20]$} \\
\hline \multicolumn{4}{|l|}{ Caregiver burden for dementia } \\
\hline Mild & 2.10 & Hours per day & \multirow{5}{*}[20]{} \\
\hline Mildly moderate & 3.58 & Hours per day & \\
\hline Moderate & 3.58 & Hours per day & \\
\hline Moderately severe & 3.76 & Hours per day & \\
\hline Severe & 5.10 & Hours per day & \\
\hline
\end{tabular}

AD: Alzheimer's disease; VaD: vascular dementia; MRI: magnetic resonance imaging; MTA: medial temporal lobe atrophy; CT: computer tomography; PET: positron emission tomography; FDG: fluorodeoxyglucose; SPECT: single photon emission computer tomography; CSF: cerebral spinal fluid.

false-negative and $11 \%$ false-positive cases versus $7 \%$ falsenegative and 3\% false-positive cases in the florbetaben group. As expected, the average life expectancy for both groups was the same. On average, patients in the florbetaben group had better clinical outcomes than the usual care group (Table 5), leading to net discounted cost savings of $\$ 11,086$ per patient over lifetime in direct medical care, $\$ 303$ in caregiver time, and $\$ 11,389$ in total cost. The majority of the cost savings resulted from reduction in costs associated with institutional care. QALYs for patients and caregivers were greater with the florbetaben group, but the differences were very small. This, nevertheless, still indicated that use of florbetaben PET 
TABLE 5: Reference-case results.

\begin{tabular}{|c|c|c|c|c|c|c|}
\hline \multirow{2}{*}{ Outcome (per patient) } & \multicolumn{3}{|c|}{ Predementia cohort $(n=320)$} & \multicolumn{3}{|c|}{ Dementia cohort $(n=680)$} \\
\hline & Usual care & Florbetaben & Net & Usual care & Florbetaben & Net \\
\hline Survival, years & 6.84 & 6.94 & 0.10 & 4.57 & 4.57 & 0.00 \\
\hline Time to confirmed diagnosis, months & 4.64 & 2.49 & -2.15 & 5.08 & 2.66 & -2.42 \\
\hline Time in predementia, years & 3.22 & 3.56 & 0.34 & N/A & N/A & N/A \\
\hline Time to institutional care, years & 5.48 & 5.72 & 0.24 & 3.17 & 3.29 & 0.12 \\
\hline \multicolumn{7}{|l|}{ Time in severity, years } \\
\hline Mild & 3.53 & 3.82 & 0.29 & 0.56 & 0.60 & 0.04 \\
\hline Mildly moderate & 0.46 & 0.44 & -0.02 & 0.77 & 0.78 & 0.01 \\
\hline Moderate & 0.48 & 0.45 & -0.03 & 0.71 & 0.71 & 0.00 \\
\hline Moderately severe & 0.42 & 0.40 & -0.01 & 0.57 & 0.56 & -0.01 \\
\hline Severe & 1.96 & 1.83 & -0.13 & 1.96 & 1.92 & -0.05 \\
\hline Caregiver time, years & 0.92 & 0.91 & -0.01 & 0.77 & 0.76 & -0.01 \\
\hline \multicolumn{7}{|l|}{ Costs (discounted) } \\
\hline Total direct medical care & $\$ 301,599$ & $\$ 289,225$ & $-\$ 12,374$ & $\$ 314,156$ & $\$ 303,070$ & $-\$ 11,086$ \\
\hline Caregiver time & $\$ 47,914$ & $\$ 47,271$ & $-\$ 643$ & $\$ 42,311$ & $\$ 42,008$ & $-\$ 303$ \\
\hline Total & $\$ 349,514$ & $\$ 336,496$ & $-\$ 13,018$ & $\$ 356,466$ & $\$ 345,077$ & $-\$ 11,389$ \\
\hline \multicolumn{7}{|l|}{ QALYs (discounted) } \\
\hline Patients & 3.53 & 3.68 & 0.15 & 1.75 & 1.78 & 0.03 \\
\hline Caregivers & 4.29 & 4.41 & 0.12 & 2.59 & 2.60 & 0.01 \\
\hline Total & 7.82 & 8.09 & 0.27 & 4.34 & 4.37 & 0.03 \\
\hline \multicolumn{7}{|l|}{ ICERs (discounted) } \\
\hline Patients & & & Dominant & & & Dominant \\
\hline Caregivers & & & Dominant & & & Dominant \\
\hline Total & & & Dominant & & & Dominant \\
\hline
\end{tabular}

QALYs: quality-adjusted life years; ICERs: incremental cost-effectiveness ratios.

Note: inconsistency may occur due to rounding.

in usual diagnostic care was a dominant strategy in the diagnosis of $\mathrm{AD}$ under this reference-case scenario.

\subsection{Deterministic Sensitivity Analyses}

3.2.1. Predementia Cohort. Figure 2 shows the top 15 model parameters with the greatest impact on total net costs. Given that the model results are very sensitive to variations in percent of patients treated in the usual diagnostic care group, treatment effect, and percent reduction in time to diagnosis confirmation by florbetaben PET, scenario analyses were conducted to understand the combined impact of the last two parameters on total net QALYs and costs in a scenario where all patients with a predementia diagnosis in the usual diagnostic care group were treated without screening. Figure 3 shows the results of the scenario analyses, indicating that treating all predementia patients without screening could be a dominant strategy as compared to using florbetaben PET to screen patients with prodromal AD for treatment if there is no reduction in the time to confirmation of dementia diagnosis with florbetaben PET. The cost effectiveness of florbetaben PET would become more favourable if predementia treatment is less effective under this scenario. This is because more patients in the usual diagnostic care group are treated than in the florbetaben group. Assuming that a treatment could reduce the risk of conversion by $25 \%$ to $50 \%$, it would require at least a $40 \%$ reduction in time to diagnosis confirmation in order for the use of florbetaben PET to be a dominant strategy under this particular scenario.

3.2.2. Dementia Cohort. The top 15 parameters influencing the net cost based on the dementia patients were similar to those observed in the predementia cohort, but the levels of significance for some model parameters were somewhat different (Figure 4). The dominance of florbetaben PET could be altered if it had no impact on time to diagnosis confirmation.

3.3. Probabilistic Sensitivity Analyses. Detailed information on how the probabilistic sensitivity analyses were performed can be viewed in Appendix D. Figures 5 and 6 show the incremental cost effectiveness planes resulting from the probabilistic sensitivity analyses based on 1,000 replications. The cost effectiveness of florbetaben PET in the diagnosis of patients with prodromal $\mathrm{AD}$ is quite uncertain as the incremental cost effectiveness ratios (ICERs) scatter across 4 different quadrants (Figure 5). Based on the results of 1,000 replications, use of florbetaben PET among the predementia patients has net QALYs gained of 0.08, ranging from a worst case of -0.67 to a best case of 1.29 , and an average net total 


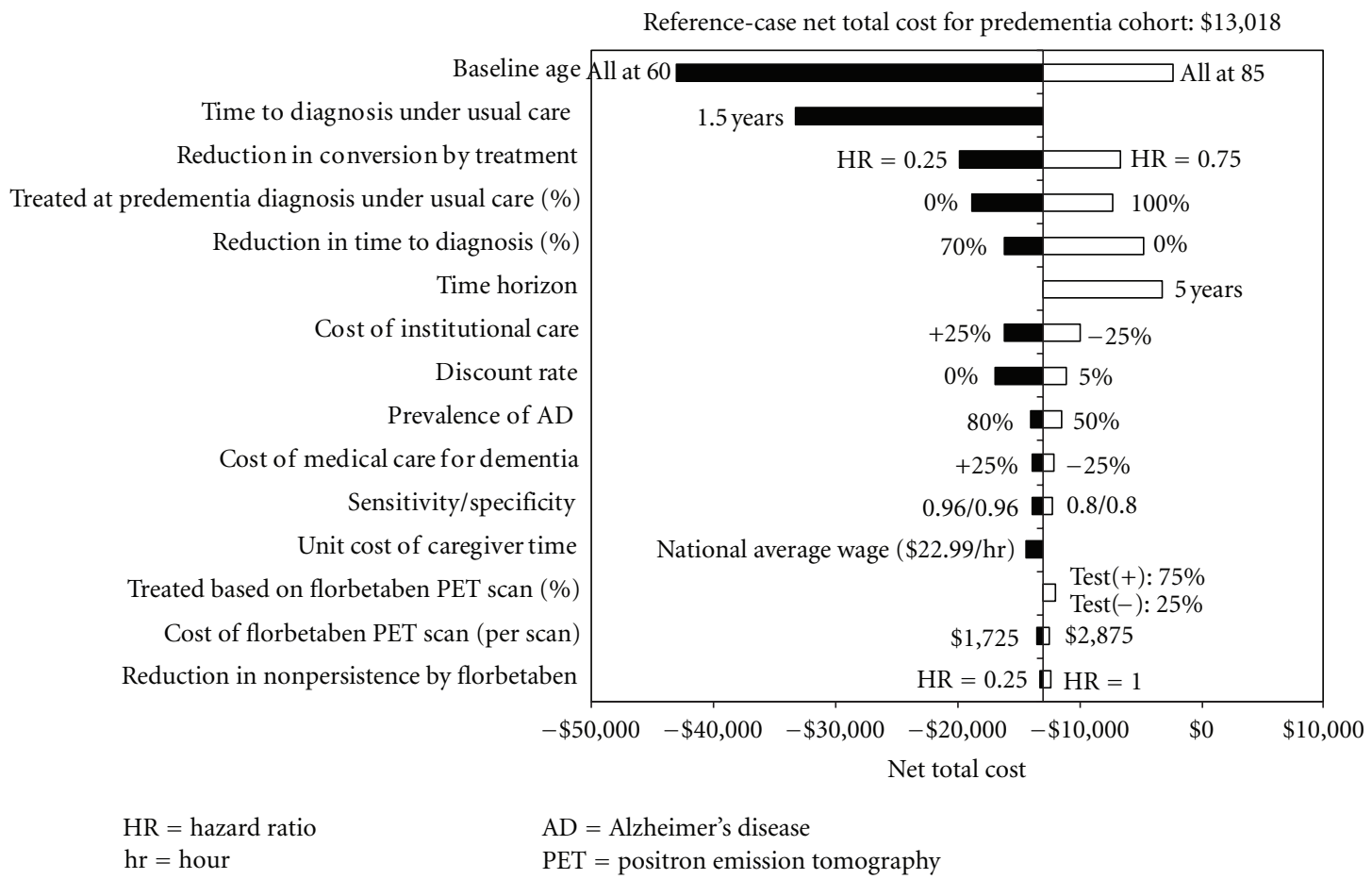

FIgURE 2: Results of univariate sensitivity analyses based on predementia cohort.

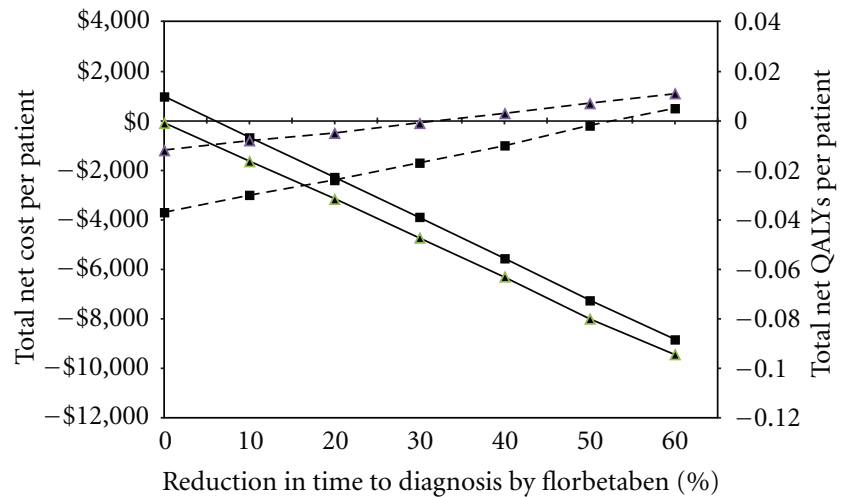

\footnotetext{
- - Total net cost $(\mathrm{HR}=0.5) \quad-\mathbf{-}-$ Total net QALYs $(\mathrm{HR}=0.5)$

$\rightarrow \mathbf{A}$ Total net cost $(\mathrm{HR}=0.75) \quad \mathbf{-} \mathbf{-}$ Total net QALYs $(\mathrm{HR}=0.75)$

$\mathrm{HR}=$ hazard ratio

QALYs = quality-adjusted life-years
}

FIGURE 3: Impact of percent reduction in time to diagnosis on net cost and quality-adjusted life-years.

cost of $-\$ 3,059$, ranging from $-\$ 101,109$ to $\$ 93,610$. Using a willingness-to-pay threshold of $\$ 50,000$ for one QALY, florbetaben PET would be considered cost effective in $58 \%$ of the replications.

Unlike the results based on the predementia patients, almost all the ICERs based on the dementia patients spread in the fourth quadrant of the incremental cost effectiveness plane (Figure 6), indicating the dominance of florbetaben
PET over usual diagnostic care. Based on the results of 1,000 replications, use of florbetaben PET among patients with dementia is associated with an average net QALY gain of 0.02 , ranging from 0.0006 to 0.09 , and a net total cost of $-\$ 9,525$, ranging from $-\$ 44,210$ to $\$ 1,117$. With a willingness-to-pay threshold of $\$ 50,000$ for one QALY, florbetaben PET is cost effective in $98 \%$ of the replications.

\section{Discussion}

To our knowledge, this is the first model to assess the cost effectiveness of a biomarker in the early diagnosis of $\mathrm{AD}$ with DES to simulate the course of disease progression from predementia to dementia phase and its clinical management from initial diagnostic work-up to treatment initiation. The greater detail underlying the DES framework allows exploration of the potential value of biomarker use in the early diagnosis of $\mathrm{AD}$, identification of major data gaps, and assessment of the uncertainty in outcomes associated with those gaps. As the model closely resembles the course of disease and its management at individual patient level, it inevitably requires richness of data to support the simulation. To deal with the data issue, we undertook a comprehensive analysis of longitudinal data from VA VISN 1 to characterize usual care pertaining to the diagnosis and treatment of $\mathrm{AD}$ and other forms of dementia in the US and to provide direct empirical estimates of various aspects of usual care of the diseases. With the use of advanced modelling technique, along with the support of comprehensive data from the VA VISN 1, this model provides a better understanding of how patients would be affected 


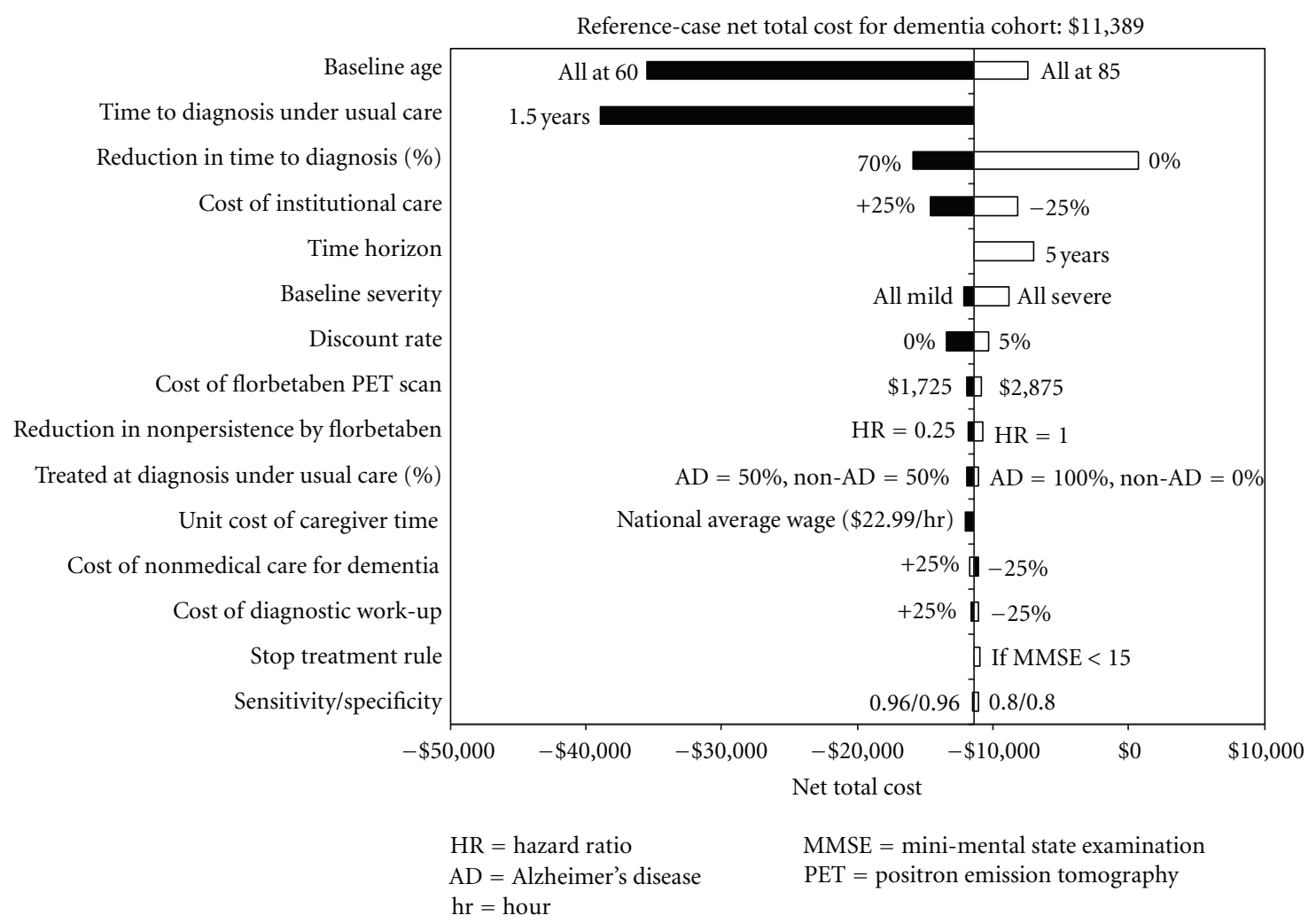

FIGURE 4: Results of univariate sensitivity analyses based on dementia cohort.

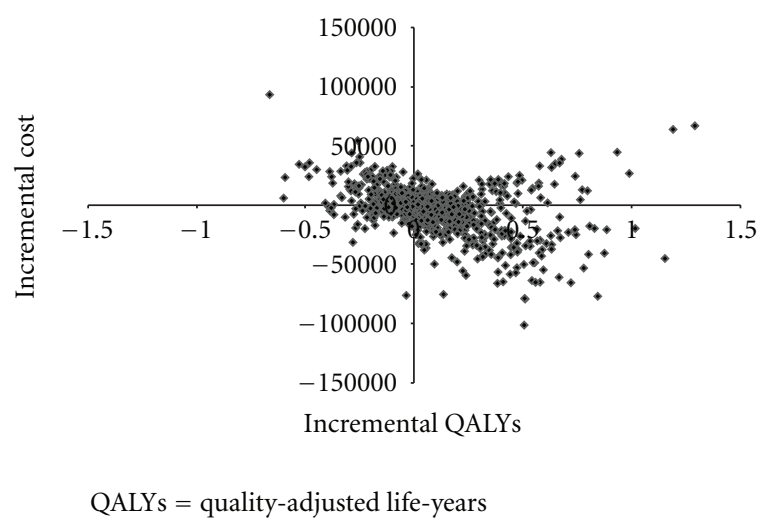

FIGURE 5: Incremental cost effectiveness plane for predementia cohort.

over time if a diagnostic biomarker like florbetaben PET tracer is used and which model parameters would have major influence on the model outcomes for specific patient groups. However, it should be noted that the results from the reference-case analysis are based on many important assumptions. Solid evidence to support or refute these assumptions is necessary before more conclusive estimates can be produced.

The reference-case scenario indicates that use of florbetaben $\mathrm{PET}$ in the diagnosis of $\mathrm{AD}$ results in both health

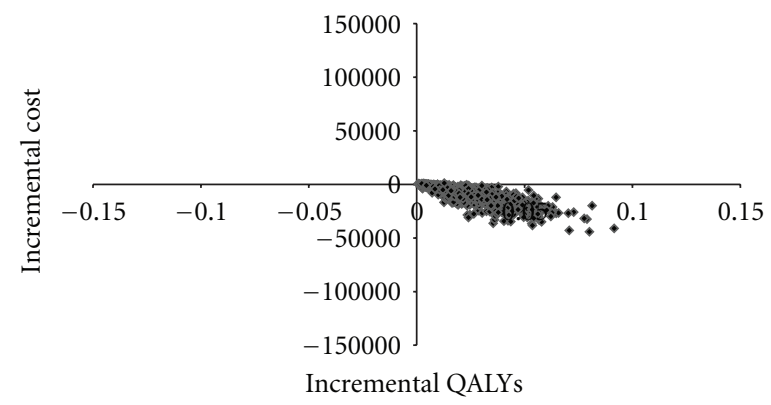

QALYs = quality-adjusted life-years

FIGURE 6: Incremental cost effectiveness plane for dementia cohort.

benefits and cost savings. The probabilistic sensitivity analyses suggest that such model outcomes are positive in the great majority of cases when florbetaben PET is used in patients with dementia but are subject to a greater uncertainty when used in patients identified with predementia. The greater uncertainty in the latter case is mainly due to lack of data on several critical model parameters. The deterministic sensitivity analyses indicate that improved accuracy of diagnosis alone would not be adequate to yield sufficient clinical benefits and cost offsets to justify the use of florbetaben PET in the diagnosis of $\mathrm{AD}$. Other clinical benefits, especially if it would shorten the time taken to confirm a dementia or 


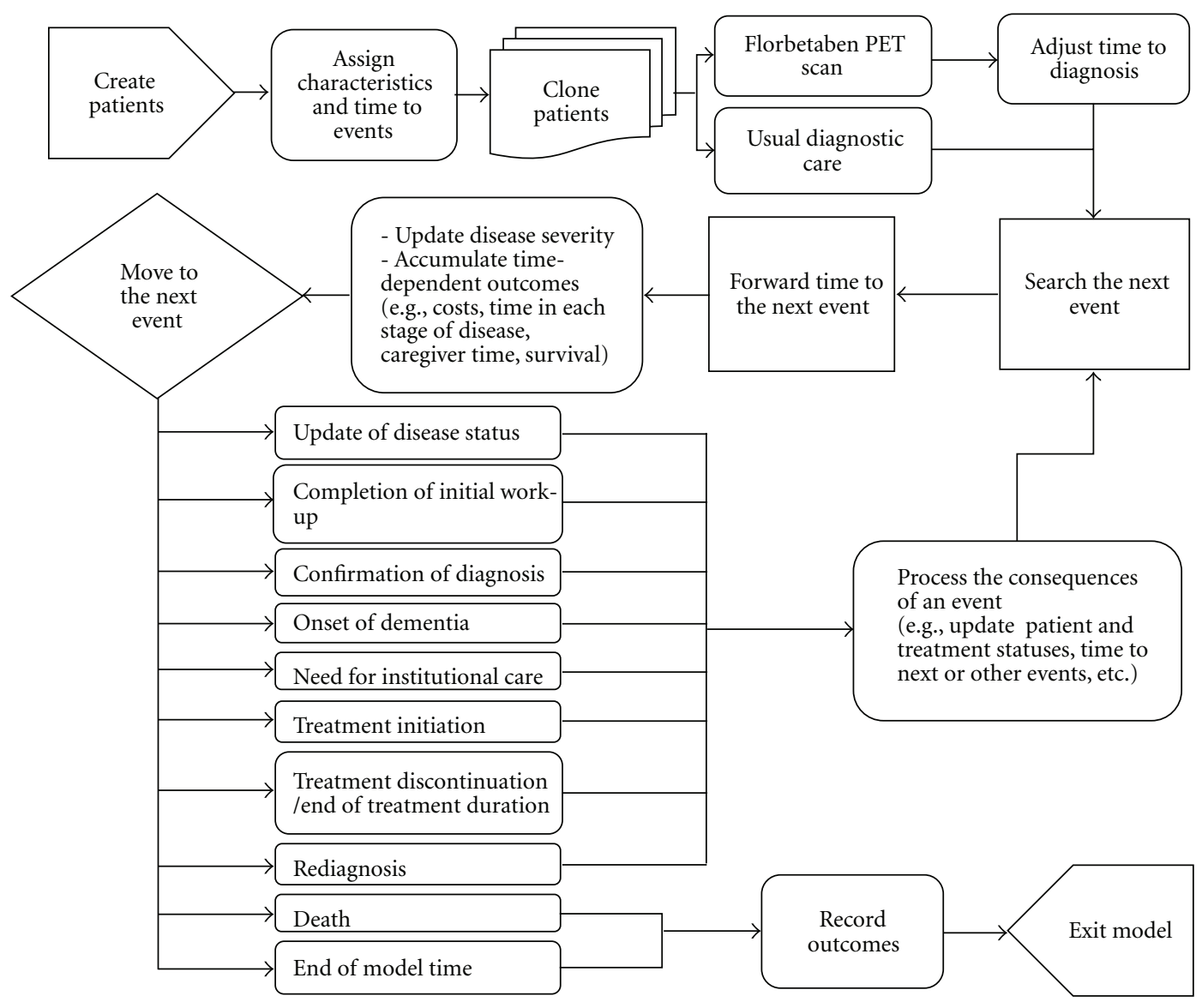

$\mathrm{PET}=$ positron emission tomography

Figure 7: Model flow.

predementia diagnosis, are needed to further support its cost effectiveness.

The reasons the reduction in time to confirmed diagnosis is so important to the cost effectiveness of florbetaben PET are not only that early diagnosis could allow appropriate dementia treatment to be initiated at an earlier stage of the disease, but also that reduction in time to diagnosis confirmation has a direct beneficial impact on time to institutional care. The risk of needing institutional care would be reduced by about $12 \%$ for every 100 -day reduction in time to diagnosis confirmation, as indicated by the analyses of the VA VISN 1 data. The causal relationship between them is still unclear. It is possible that early confirmation of diagnosis would allow patients and their family members to plan ahead and make needed adjustments to keep patients living independently as long as possible before they are sent to longterm-care facilities. Given that institutional care is costly, any minor delay to institutional care would have a meaningful impact on offsetting the cost of the scan. Data from a survey study, conducted alongside the florbetaben PET phase IIA trial [41] seem to also suggest that use of florbetaben PET can reduce the time to diagnosis confirmation. The survey shows that directly visualizing and evaluating a patient's amyloid burden in vivo highly increases the confidence of physicians in making their final diagnosis, suggesting that physicians are very likely to shorten the diagnostic work-up by eliminating additional examinations and "watchful" waiting period before sufficient symptoms are observed and consequently initiate appropriate treatment at an earlier stage. This could have a substantial positive impact on health and resource utilization benefits as there were approximately $72 \%$ and $23 \%$ of patients with predementia and AD diagnoses in the VA VISN 1 study cohort, respectively, who did not receive any dementia medication at confirmation until a median time of 42 and 28 months after confirmation. Use of florbetaben may shorten the delay to receipt of appropriate care substantially and result in better clinical and economic outcomes.

Three additional important findings from the present analyses are worth mentioning as they may have important implications for future economic assessment of the florbetaben PET tracer in the early diagnosis of AD. First, a large discrepancy on time to confirmed diagnosis was found from the VA VISN 1 data when different approaches, that is, analysis of administrative data versus chart review of a subset of the study cohort, were used to quantify this duration. The estimated duration based on the analyses of the VA VISN 1 
data may better capture the time taken to confirm a dementia diagnosis from the initial office visit because a diagnosis code would normally be recorded to represent the main complaint for a particular office visit. Yet, the estimated duration of 1.5 years based on the review of medical records should be a good proxy for the time to confirmation of diagnosis from the early signs and symptoms of $\mathrm{AD}$ as these could be recorded in the medical charts during the office visits for other medical problems. The implication of this discrepancy seems to pose a great opportunity for florbetaben PET tracers to identify patients with prodromal $\mathrm{AD}$ even at a much earlier stage if clinicians know when to use them. This would have a substantial, favourable impact on the cost effectiveness of florbetaben PET tracers.

Second, our deterministic sensitivity analyses show that younger patients would have a greater gain in net cost savings and QALYs from the use of florbetaben PET. This is due to a longer life expectancy in this population. In order to treat patients with prodromal $\mathrm{AD}$ or $\mathrm{AD}$ dementia at a younger age, screening general populations at younger ages seems to be a reasonable strategy. Although screening the general population for $\mathrm{AD}$ is not the focus of this assessment, the significant gain in economic and clinical benefits in the younger group from our analyses does suggest a promising possibility to support such an application for florbetaben PET tracers. This could also have valuable benefits to some of the patients who choose to know their disease propensity as early as possible despite the absence of effective treatments during the preclinical or predementia phase as it allows them to plan ahead with their life for personal and financial reasons $[42,43]$.

Third, as there is still no convincing evidence to support that treatment with cholinesterase inhibitors would yield any survival benefit, this model assumes that survival is independent of treatment effect, consistent with the assumption made in other published models $[25,26]$. It is nevertheless important to highlight that if treatment does result in improved survival due to reduction in the rate of disease progression, use of florbetaben PET would yield more QALYs gained, but at the same time would result in higher overall costs, especially for long-term care. As future disease modifying treatments for $\mathrm{AD}$ may extend survival, this may have an important impact on the economic value of florbetaben PET and should be considered in future economic assessment of this technology.

The present model has several major limitations. First, because the current analyses are mainly based on the data from the New England VA Healthcare System, the findings from this analysis may not be generalizable to patients in other regions of the VA Healthcare System, as well as in other healthcare systems outside of the VA system. Second, an external validation of this model to examine how well the model can predict the results observed in other studies has not yet been conducted due to lack of an appropriate external data source. However, the results of key model components, including disease progression during the dementia phase and time to clinical events shown in Table 2, were validated against the results from their respective data sources. A more complete external validation should be conducted when appropriate data source becomes available. Third, consistent with many other modelling studies in this therapeutic area $[25,26]$, continued treatment with dementia medication after 1 year was assumed to have a maintenance function only and no further treatment benefits in terms of delaying disease progression. Fourth, the model assumes that all patients who present to their doctor for memory complaints have some type of dementia. This may not be completely true as memory complaints or cognitive problems could be caused by other health problems, such as depression. Inclusion of these patients who in fact have no dementia may have some impact on the model results, depending on the prevalence of these conditions. Finally, the model, for the purpose of simplicity, assumed that all non-AD patients with a negative result at the end of diagnostic work-up would have their diagnosis correctly confirmed. This might have underestimated the benefits of florbetaben PET as it helps rule out $\mathrm{AD}$ as the underlying cause with a greater degree of certainty compared to other diagnostic tools, which may consequently help identify the true cause for non-AD dementia.

\section{Conclusions}

This economic model provides a comprehensive framework to explore the potential clinical and economic value of florbetaben PET in the early diagnosis of $\mathrm{AD}$ among patients who present to their physicians' office for the first time due to cognitive complaints, to identify key value drivers as well as potential data gaps. Our exploratory analyses suggest that florbetaben PET has the potential to be a valuable tool in the diagnosis of $\mathrm{AD}$ as it would improve the health benefits of patients (with dementia as well as predementia) and their caregivers at a lower cost under certain scenarios. While the findings from the analyses to a large extent are supported by the data from a large longitudinal database and published literature, they rest also on many key assumptions and are subject to great uncertainty. Data on how the technology would impact clinical decision making and outcomes, such as time to confirmation of diagnosis, treatment uptake, and treatment persistency, will be needed to further substantiate its value.

\section{Appendices}

\section{A. Model Flow}

A simplified flow diagram showing how patients are simulated in the model is displayed above. At the beginning of the simulation, the model creates 1,000 patients with different types of dementia, based on the prevalence of dementia for each type specified by the user, and assigns patient and disease characteristics conditional on their underlying cause. These characteristics include age, gender, race, baseline scores for Mini-Mental State Examination (MMSE), Neuropsychiatric Inventory (NPI), activities of daily living (ADL), instrumental activities of daily living (IADL), location of care (either home or institutional care), 
use of antipsychotics, comorbidities (i.e., chronic kidney disease, cardiovascular disease, diabetes, and hypertension), and caregiver's gender and age. These characteristics are used to predict the rate of disease progression and other model outcomes, such as time to death, time to institutional care, time to confirmation of diagnosis, costs of care, and health utilities. After the assignments of baseline patient characteristics and event times, each patient is cloned; one clone is assigned to the usual diagnostic care group and the other to the florbetaben group. The cloning step used in the simulation resembles a perfect randomization where both groups are comprised of exactly the same patients. For those assigned to the florbetaben group, their time to confirmation of diagnosis is updated based on a percent reduction in time to diagnosis specified by the user. Then, all patients are sent to the "search next event" module, where the next event for each patient is identified based on the event with the shortest time to occur. There are a total of 10 events conceptualized in this model, as shown in diagram above. After identifying the next event for a patient, the model then fast-forwards its clock to the next event time. Before the event is processed for its related consequences, such as updates of patient and treatment statuses and time to next event, all timedependent outcomes-including survival, quality-adjusted life-years (QALYs), costs of care, caregiver time, time alive at each stage of the disease, and time spent in institutional care-are tallied and accumulated. After processing the consequences of the event, the patient proceeds to the "search next event" module again, and the same process is repeated until the patient dies or the model time ends see Figure 7.

\section{B. Database Analysis}

The primary data source used to populate this model was based on the administrative databases (clinical, laboratory, and pharmacy databases) from New England Veterans Healthcare System (VISN 1) from January 1, 2002 through December 31, 2009 (fiscal years 2002-2009). The New England region consists of eight Veterans Administration (VA) Medical Centers and affiliated clinics providing inpatient and outpatient medical care. The VISN 1 pharmacy files were obtained from Information Resource Management, Boston, MA. ICD-9-CM diagnoses and laboratory data were captured by accessing the VA National Patient Care and Decision Support Systems administrative databases (Patient Treatment File and Outpatient Care File) located at the Austin Automation Center, Austin, TX. All database analyses were conducted at the Massachusetts Veterans Epidemiology Research and Information Center (MAVERIC), VA Boston Healthcare System, Boston, MA. The study was approved by the Institutional Review Board of the VA Boston Healthcare System.

Specific analyses from VISN 1 were performed to obtain the majority of the model inputs, including the following: (1) prevalence of dementia for each type and baseline patients and disease characteristics by dementia diagnosis, (2) time to confirmation of diagnosis from the initial visit, (3) resource use during the diagnostic work-up period, (4) proportion of the patients treated with cholinesterase inhibitors or memantine at time of diagnosis confirmation by dementia diagnosis, (5) time to treatment initiation if not treated at time of confirmation, (6) time to treatment discontinuation if treated, (7) time to conversion to the dementia phase for patients with predementia, and (8) time to institutional care. The study cohort for these analyses was based on 2,783 patients who were confirmed with a dementia $(n=1,882)$ or predementia $(n=901)$ diagnosis within two years since the initial visit. The maximum two-year time to diagnosis confirmation was used to exclude those cases that may not actually have any dementia. The study cohort was selected from more than 19,000 subjects in the New England VA Healthcare System between January 1, 2002 and December 31, 2009 and who had one of the following dementia diagnoses Alzheimer disease (AD), senile and presenile of dementia of the $\mathrm{AD}$ type, vascular dementia $(\mathrm{VaD})$, frontotemporal dementia (FTD), Lewy body dementia (LBD), mild cognitive impairment (MCI), memory loss, and cognitive deficits of cerebrovascular disease (CD-CVD). Additional inclusion criteria included patients at least 55 years of age at time of first dementia diagnosis, a 12-month baseline period in the VA system free of any dementia diagnosis prior to the first dementia diagnosis, and no record of cholinesterase inhibitors or memantine during the baseline period. Specific types of dementia diagnosis were confirmed if patients met one of the following two criteria: (1) at least two identical dementia ICD-9 diagnoses given by a recognized specialist, separated by a period of at least 30 days, and (2) at least one ICD-9 diagnosis of dementia accompanied by a recognized dementia medication, including donepezil, rivastigmine, galantamine, or memantine. Descriptive statistical analyses and derivations of parametric equations to predict time to events based on patient characteristics were performed.

Among the patients with a confirmed diagnosis $(n=$ $2,783), 68 \%$ of them were confirmed with a dementia diagnosis and $32 \%$ with a predementia diagnosis. For those with a dementia diagnosis, $67 \%$ had a confirmed diagnosis of $\mathrm{AD}$ or mixed $\mathrm{AD}, 28 \%$ had a $\mathrm{VaD}$, and the remaining $5 \%$ had other dementia diagnoses, such as LBD, FTD, and mixed non-AD. For those diagnoses with predementia, $65 \%$ were assumed to have prodromal $\mathrm{AD}$, which was estimated based on a chart review of a subset of these patients. The mean age at initial diagnosis was about 78 years for patients with predementia and 82 years for patients with dementia. Comorbidities were highly prevalent among the study cohort due to their advanced age, with about $20 \%$ with a CVD, $27 \%$ with diabetes, $47 \%$ with coronary heart disease, $80 \%$ with hypertension, and $5 \%$ on antipsychotics at baseline.

\section{Cost Inputs}

C.1. Diagnostic Work-Up. The costs of diagnostic work-up, including a series of office visits to primary care physicians and specialists, lab tests, and outpatient clinic visits, were estimated based on the time taken to confirm a diagnosis from the initial office visit. Data for healthcare resource use during the diagnostic work-up period were obtained for 
each dementia diagnosis from the VA VISN 1 data and were translated into costs by applying the unit costs from the Centers for Medicare \& Medicaid Services (CMS) [16-18]. The estimated costs of diagnostic work-up for each diagnosis per year are shown in Table 4 of the main text.

C.2. Imaging and Biomarker Tests. The costs of brain imaging and biomarker analyses, a one-time cost based on the distribution of diagnostic algorithms assigned, were estimated using the cost data from CMS hospital outpatient fee schedule [16] (Table 4). The cost of florbetaben PET was set at $\$ 2,300$ per test in the base-case analysis, which was suggested by the manufacturer of florbetaben.

C.3. Dementia Medication. Unit costs for dementia medications were based on the average wholesale price reported in the Red Book [19] (Table 4). The daily costs for these drugs were estimated based on their recommended dose and usage from the licensed labels.

C.4. Medical and Nonmedical Care for Predementia and Dementia. Costs of care were separated into costs of medical and nonmedical care. For patients with $\mathrm{AD}$, these costs were obtained from a longitudinal study which followed 172 patients with probable $\mathrm{AD}$ for 4 years to examine the effects of patient dependence, measured by the Dependence Scale, on the following: (1) medical care costs including hospitalizations, outpatient treatments and procedures, and assistive devices; (2) nonmedical care costs, including overnight respite care, adult day care, and home healthcare; (3) informal caregiving time, including time used for ADL and for supervision [20]. As these outcomes of interest were reported in relation to the Dependence Scale (range $=0$ 15; higher scores indicate greater dependence), they were mapped onto the MMSE scores using the data from the same study to estimate the costs of medical and nonmedical care, as well as caregiver time, by level of severity, defined by MMSE scores. Table 4 shows the costs of medical and nonmedical care and the amount of caregiver time by each level of severity for patients with $\mathrm{AD}$. As these costs were measured in 2005 values, they were inflated to 2011 values using the medical services component of the Consumer Price Index. The costs of care for patients with non-AD dementia were also estimated based on patients' levels of severity. The annual costs of care for patients with $\mathrm{VaD}$ and other non-AD were estimated by increasing 1.83 and 1.37 times the costs for patients with $\mathrm{AD}$, respectively. These ratios were obtained from a study comparing the healthcare costs of communitydwelling patients with $\operatorname{VaD}(n=678)$ and other non-AD dementia $(n=957)$ to patients with $\mathrm{AD}(n=1,722)$ using Medicare HMO data during 1999-2002 [21]. The amount of caregiver time by level of severity for patients with non-AD was assumed to be the same as that for patients with AD due to lack of available data.

The cost of managing predementia in the US setting was not identified from the literature search. Although some studies were found for other countries $[38,44]$, they were not used in the analyses, as healthcare utilization patterns and costs vary significantly across countries. Thus, the average cost based on the Dependence Scale of 0-1 obtained from the study by Zhu and colleagues [20] was used to estimate the cost of care and caregiver time for patients with predementia (Table 4) [20].

\section{Analyses}

Model outcomes for the base-case analysis included percent of patients misdiagnosed, time to diagnosis confirmation, number of predementia patients progressing to dementia, life-years, time alive at each severity stage, percent of patients needing institutional care, time to institutional care, caregiver time, costs, and QALYs for patients and caregivers. Deterministic sensitivity analyses, including one-way, subgroup, and scenario analyses, were performed to assess how the model outcomes vary in relation to changes in model parameters. Finally, in order to account for uncertainties from multiple key parameters, probabilistic sensitivity analyses were performed by simultaneously varying multiple parameters under the following assumptions. First, for the inputs which have no or little prior data to support, uniform distributions were used as it is a more conservative assumption. These parameters included the following: (1) sensitivity (80\% for the lower bound-96\% for the upper bound) and specificity (80-96\%) of florbetaben PET, (2) percent reduction $(0-100 \%)$ in time to diagnosis confirmation by florbetaben PET, (3) percent reduction (0-100\%) in risk of conversion to dementia phase by treatment, and (4) percent reduction $(0-100 \%)$ in treatment discontinuation by florbetaben PET. Second, for model inputs with prior data to support, beta distributions were used for categorical variables and normal distributions within 2 standard errors of the mean as the upper and lower bounds were used for continuous variables. For some of the parameters included in the probabilistic sensitivity analyses, standard errors were available from the parameter source data and thus used to measure parameter uncertainties. Where a standard error was not available for a selected parameter, we used $25 \%$ of the mean as an assumed standard error. Parameters included in the probabilistic sensitivity analyses and assumed to be beta distributed were as follows: (1) percents of the predementia and dementia patients treated at time of confirmation under usual diagnostic care, (2) ratios of cost of care for $\mathrm{AD}$ versus $\mathrm{VaD}$ and non-AD, and (3) patient and caregiver utilities for predementia patients. Parameters assumed to be normally distributed included the following: (1) all predictor coefficients for the parametric equations, including time to diagnosis, time to treatment initiation, time to treatment discontinuation, and time to institutional care; (2) coefficients for the treatment effects on rates of change in MMSE, NPI, ADL, and IADL over time; (3) hazard ratios of death (with log transformation) compared to general population for predementia, $\mathrm{AD}$, and no-AD; (4) predictor coefficients for patient and caregiver utility equations for dementia patients; (5) cost of dementia medications; (6) costs of medical and nonmedical care for predementia and 
dementia; (7) cost of institutional care; (8) caregiver burden by severity level.

\section{Acknowledgments}

All authors participated in the conception and implementation of the model, data analyses for obtaining model inputs and analyses of the simulation, and writing of the paper. S. Guo, D. Getsios, L. Hernandez, and S. Lanes are employees of United BioSource Corporation (UBC), a consultancy that has also received grants for other unrelated research from various pharmaceutical companies. E. Lawler and K. Cho are employees of the Massachusetts Veteran's Research and Information Center, which is supported by the Veterans Affairs Cooperative Studies Program. A. Altincatal is an employee of Boston VA Research Institute, Inc. M. Blankenburg is an employee of Bayer Healthcare Pharmaceuticals, located in Berlin, Germany. This work was sponsored by Bayer Healthcare Pharmaceuticals, the manufacturer of florbetaben tracers. The authors thank Jens Kuhlmann, Martin Pessel, and Andreas Kramell for critically reading the paper.

\section{References}

[1] J. L. Cummings, “Alzheimer's disease," The New England Journal of Medicine, vol. 351, no. 1, pp. 56-67, 2004.

[2] K. D. Kochanek, J. Xu, S. L. Murphy et al., "Deaths: preliminary data for 2009," National Center for Health Statistics, vol. 59, no. $4,2011$.

[3] A. Wimo, B. Winblad, and L. Jönsson, "The worldwide societal costs of dementia: estimates for 2009," Alzheimer's and Dementia, vol. 6, no. 2, pp. 98-103, 2010.

[4] J. Birks, "Cholinesterase inhibitors for Alzheimer's disease," Cochrane Database of Systematic Reviews, no. 1, Article ID CD005593, 2006.

[5] R. A. Hansen, G. Gartlehner, A. P. Webb, L. C. Morgan, C. G. Moore, and D. E. Jonas, "Efficacy and safety of donepezil, galantamine, and rivastigmine for the treatment of Alzheimer's disease: a systematic review and meta-analysis," Clinical Interventions in Aging, vol. 3, no. 2, pp. 211-225, 2008.

[6] S. Banarjee and R. Wittenberg, "Clinical and cost effectiveness of services for early diagnosis and intervention in dementia," International Journal of Geriatric Psychiatry, vol. 24, no. 7, pp. 748-754, 2009.

[7] D. Getsios, S. Blume, K. J. Ishak, G. Maclaine, and L. Hernández, "An economic evaluation of early assessment for Alzheimer's disease in the United Kingdom," Alzheimer's and Dementia, vol. 8, no. 1, pp. 22-30, 2012.

[8] M. Löppönen, I. Räiha, R. Isoaho, T. Vahlberg, and S. L. Kivelä, "Diagnosing cognitive impairment and dementia in primary health care- - a more active approach is needed," Age and Ageing, vol. 32, no. 6, pp. 606-612, 2003.

[9] N. A. Ranginwala, L. S. Hynan, M. F. Weiner, and C. L. White, "Clinical criteria for the diagnosis of alzheimer disease: still good after all these years," American Journal of Geriatric Psychiatry, vol. 16, no. 5, pp. 384-388, 2008.

[10] D. H. Taylor Jr., T. Østbye, K. M. Langa, D. Weir, and B. L. Plassman, "The accuracy of medicare claims as an epidemiological tool: the case of dementia revisited," Journal of Alzheimer's Disease, vol. 17, no. 4, pp. 807-815, 2009.

[11] L. M. Bloudek, D. E. Spackman, M. Blankenburg et al., "Review and meta-analysis of biomarkers and diagnostic imaging in Alzheimer's disease," Journal of Alzheimer's Disease, vol. 26, no. 4, pp. 627-645, 2011.

[12] B. Dubois, H. H. Feldman, C. Jacova et al., "Revising the definition of Alzheimer's disease: a new lexicon," The Lancet Neurology, vol. 9, no. 11, pp. 1118-1127, 2010.

[13] H. Barthel, H. J. Gertz, S. Dresel et al., "Cerebral amyloid$\beta$ PET with florbetaben (18F) in patients with Alzheimer's disease and healthy controls: a multicentre phase 2 diagnostic study," The Lancet Neurology, vol. 10, no. 5, pp. 424-435, 2011.

[14] H. Barthel, J. Luthardt, G. Becker et al., "Individualized quantification of brain $\beta$-amyloid burden: results of a proof of mechanism phase 0 florbetaben PET trial in patients with Alzheimer's disease and healthy controls," European Journal of Nuclear Medicine and Molecular Imaging, vol. 38, no. 9, pp. 1702-1714, 2011.

[15] V. L. Villemagne, K. Ong, R. S. Mulligan et al., "Amyloid imaging with (18)F-florbetaben in Alzheimer disease and other dementias," Journal of Nuclear Medicine, vol. 52, no. 8, pp. 1210-1217, 2010.

[16] Center for Medicare and Medicaid Services, Hospital Outpatient File PPS Payment Rate, 2011.

[17] Center for Medicare and Medicaid Services, Clinical Diagnostic Laboratory Fee Schedule, 2011.

[18] Center for Medicare and Medicaid Services, CMS Physician Fee Schedule, 2011.

[19] Red Book, Red Book For Windows 2011,060, 2011.

[20] C. W. Zhu, C. Leibman, T. McLaughlin et al., "Patient dependence and longitudinal changes in costs of care in Alzheimer's disease," Dementia and Geriatric Cognitive Disorders, vol. 26, no. 5, pp. 416-423, 2008.

[21] J. Hill, H. Fillit, S. N. Shah, M. C. del Valle, and R. Futterman, "Patterns of healthcare utilization and costs for vascular dementia in a community-dwelling population," Journal of Alzheimer's Disease, vol. 8, no. 1, pp. 43-50, 2005.

[22] Center for Medicare and Medicaid Services, "Healthcare financing review," in 2010 Statistical Supplement, 2010.

[23] US Department of Labor, Federal minimal wage, 2011, http://www.dol.gov.

[24] J. J. Caro, "Pharmacoeconomic analyses using discrete event simulation," PharmacoEconomics, vol. 23, no. 4, pp. 323-332, 2005.

[25] D. Getsios, S. Blume, K. J. Ishak, and G. D. H. Maclaine, "Cost effectiveness of donepezil in the treatment of mild to moderate Alzheimer's disease: a UK evaluation using discreteevent simulation," PharmacoEconomics, vol. 28, no. 5, pp. 411$427,2010$.

[26] S. Guo, L. Hernandez, R. Wasiak, and M. Gaudig, "Modelling the clinical and economic implications of galantamine in the treatment of mild-to-moderate Alzheimer's disease in Germany," Journal of Medical Economics, vol. 13, no. 4, pp. 641-654, 2010.

[27] J. Lipscome, M. C. Weinstein, and G. W. Torrance, "Time preference," in Cost-Effectiveness in Health and Medicine, M. R. Gold, J. E. Siegel, L. B. Russell et al., Eds., Oxford University Press, New York, 1996.

[28] National Institute for Health and Clinical Excellence, "Donepezil, galantamine, rivastigmine (review) and memantine for the treatment of Alzheimer's disease," 2006, http://www.nice.org.uk/. 
[29] M. Ganguli, H. H. Dodge, C. Shen, and S. T. DeKosky, "Mild cognitive impairment, amnestic type: an epidemiologic study," Neurology, vol. 63, no. 1, pp. 115-121, 2004.

[30] B. L. Plassman, K. M. Langa, G. G. Fisher et al., "Prevalence of dementia in the United States: the aging, demographics, and memory study," Neuroepidemiology, vol. 29, no. 1-2, pp. 125132, 2007.

[31] J. Schultz and V. Joish, "Costs associated with changes in antidepressant treatment in a managed care population with major depressive disorder," Psychiatric Services, vol. 60, no. 12, pp. 1604-1611, 2009.

[32] C. Ballard, J. O’Brien, C. M. Morris et al., “The progression of cognitive impairment in dementia with Lewy bodies, vascular dementia and Alzheimer's disease," International Journal of Geriatric Psychiatry, vol. 16, no. 5, pp. 499-503, 2001.

[33] H. Hanyu, T. Sato, K. Hirao, H. Kanetaka, H. Sakurai, and T. Iwamoto, "Differences in clinical course between dementia with Lewy bodies and Alzheimer's disease," European Journal of Neurology, vol. 16, no. 2, pp. 212-217, 2009.

[34] K. Rascovsky, D. P. Salmon, A. M. Lipton et al., "Rate of progression differs in frontotemporal dementia and Alzheimer disease," Neurology, vol. 65, no. 3, pp. 397-403, 2005.

[35] M. M. Williams, C. Xiong, J. C. Morris, and J. E. Galvin, "Survival and mortality differences between dementia with Lewy bodies vs Alzheimer disease," Neurology, vol. 67, no. 11, pp. 1935-1941, 2006.

[36] K. D. Kochanek, J. Xu, S. L. Murphy et al., 2009 US Life Table, March, 2011.

[37] R. S. Wilson, N. T. Aggarwal, L. L. Barnes, J. L. Bienias, C. F. Mendes De Leon, and D. A. Evans, "Biracial population study of mortality in mild cognitive impairment and Alzheimer disease," Archives of Neurology, vol. 66, no. 6, pp. 767-772, 2009.

[38] L. Jönsson, N. Andreasen, L. Kilander et al., "Patient- and proxy-reported utility in Alzheimer disease using the EuroQoL," Alzheimer Disease and Associated Disorders, vol. 20, no. 1, pp. 49-55, 2006.

[39] C. K. Andersen, K. U. Wittrup-Jensen, A. Lolk, K. Andersen, and P. Kragh-Sørensen, "Ability to perform activities of daily living is the main factor affecting quality of life in patients with dementia," Health and Quality of Life Outcomes, vol. 2, article 52, 2004.

[40] M. Ekman, J. Berg, A. Wimo, L. Jönsson, and C. McBurney, "Health utilities in mild cognitive impairment and dementia: a population study in Sweden," International Journal of Geriatric Psychiatry, vol. 22, no. 7, pp. 649-655, 2007.

[41] C. G. Schipke, O. Peters, I. Heuser et al., "Impact of betaamyloid-specific Florbetaben PET imaging on early diagnosis and patient management in Alzheimer's disease," Dementia and Geriatric Cognitive Disorders, vol. 33, no. 6, pp. 416-422, 2012.

[42] P. J. Neumann, J. T. Cohen, J. K. Hammitt et al., "Willingnessto-pay for predictive tests with no immediate treatment implications: a survey of US residents," Health Economics, vol. 21, no. 3, pp. 238-251, 2012.

[43] P. J. Neumann, J. K. Hammitt, C. Mueller et al., "Public attitudes about genetic testing for alzheimer's disease," Health Affairs, vol. 20, no. 5, pp. 252-264, 2001.

[44] M. Luppa, S. Heinrich, H. Matschinger, H. Sandholzer, M. C. Angermeyer, H. H. Konig et al., "Direct costs associated with depression in old age in Germany," Journal of Affective Disorders, vol. 105, no. 1-3, pp. 195-204, 2008. 


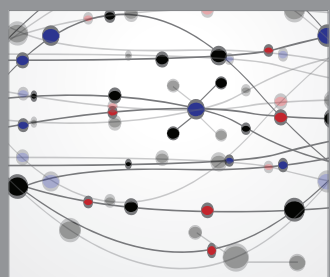

The Scientific World Journal
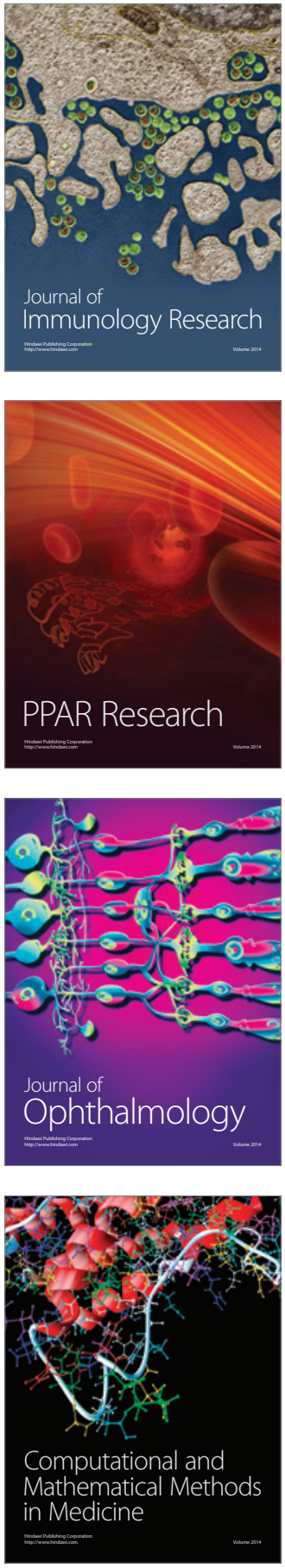

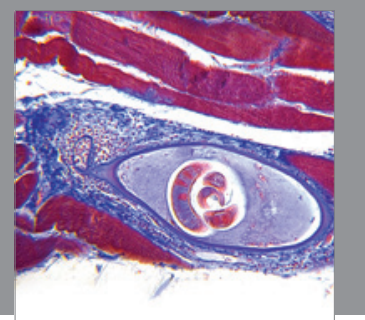

Gastroenterology

Research and Practice
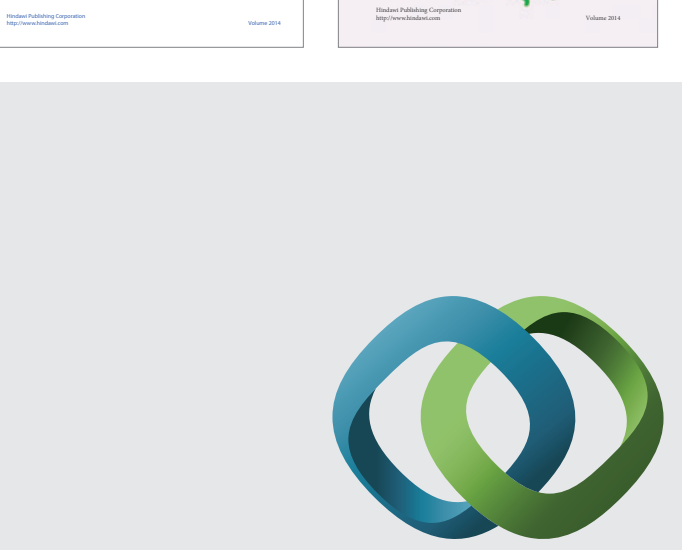

\section{Hindawi}

Submit your manuscripts at

http://www.hindawi.com
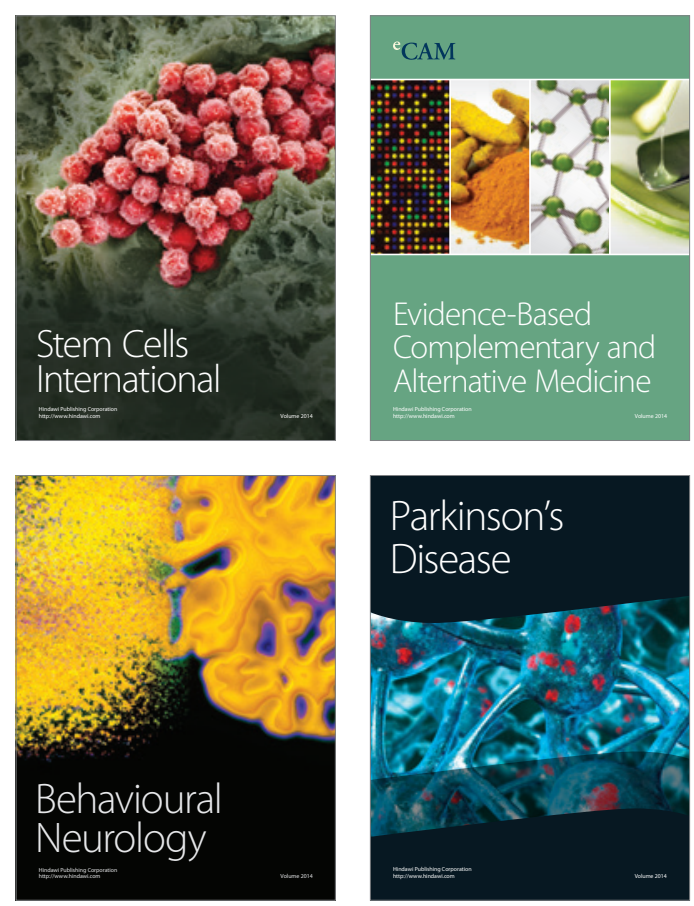

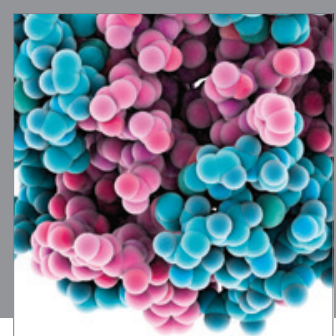

Journal of
Diabetes Research

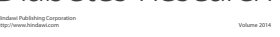

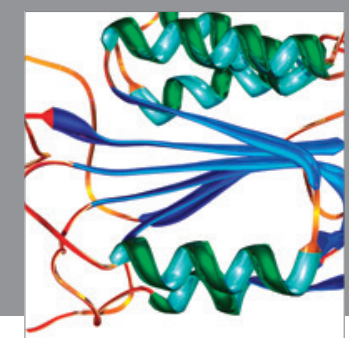

Disease Markers
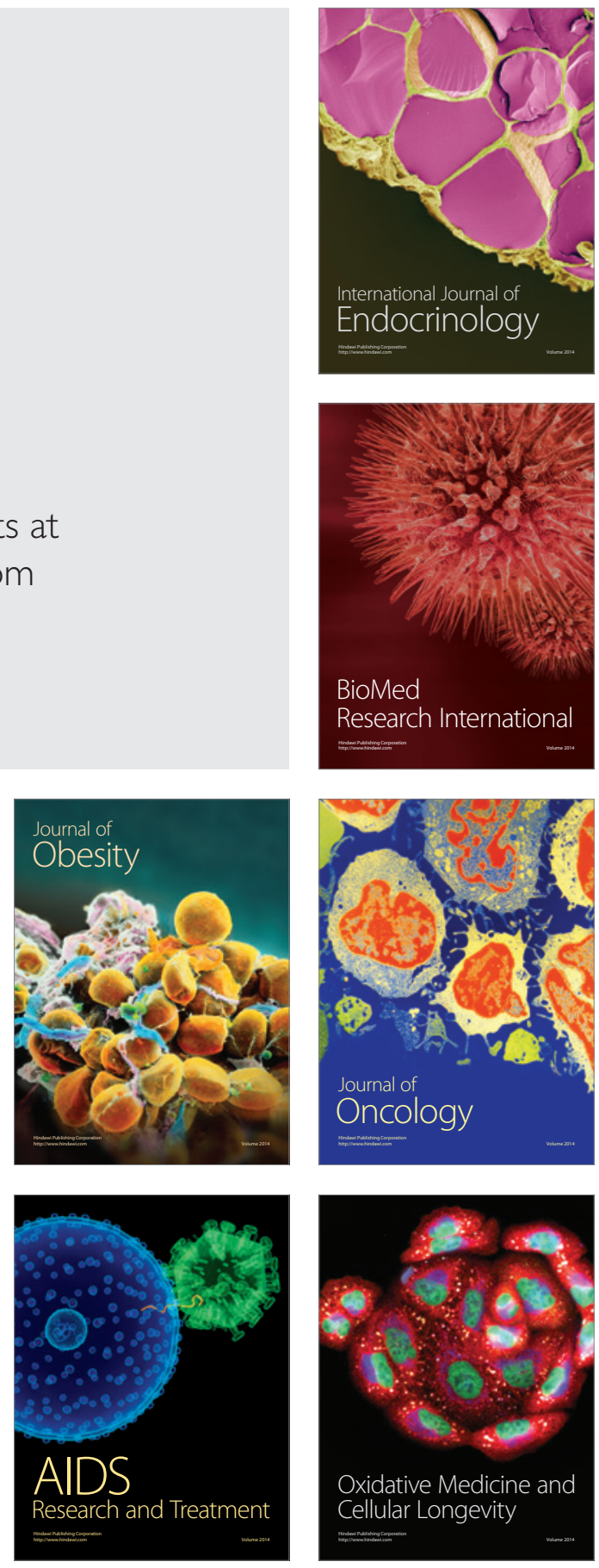